\title{
Numerical Modeling of Stress Corrosion Cracking of Polymers
}

\author{
Hanxiao Ge, Jialiang Le, Susan C. Mantell
}

\begin{abstract}
In this paper, a unified chemo-mechanical model is developed to simulate the stress corrosion cracking (SCC) of high density polyethylene (HDPE) in a chlorinated environment. The model is comprised of three components, each capturing a critical aspect of SCC. A chemical kinetics and diffusion model is used to simulate the reactions and migration of chemical substances. The fracture behavior of HDPE is captured by using a cohesive crack model, in which the cohesive properties are considered to be dependent on the extent of the chemical degradations. The time-dependent creep behavior of the bulk HDPE material is described by an elastic-viscoplastic constitutive model. This chemo-mechanical model is numerically implemented for finite element analysis of SCC of HDPE structural components. The simulations show two different failure mechanisms depending on the applied stress level: at high stresses, the failure is primarily due to the excessive plastic deformation whereas at low stresses the chemical reactions and diffusion are the dominant factors leading to failure. In addition, the examination of detailed crack growth kinetics reveals that at low stress levels the disinfectant concentration has a significant effect on the crack growth behavior depending on the relative dominance between the chemical reaction and diffusion processes.
\end{abstract}

\section{Introduction}

Polymers are increasingly used for structural applications owing to their corrosion resistance and low cost compared with metals. Typical applications of polymers for civil infrastructure (without reinforcing fibers) include: geomembranes, geogrids, pipelines for water and natural gas delivery, corrugated drainage pipes and sanitary pipes. Longevity of polymeric components is a critical aspect of these infrastructure. However, exposure to an oxidative environment could lead to premature failure of the polymeric components. For example, geomembranes have been widely used to contain hazardous and waste liquid/solid and its longterm structural integrity is of great concern to designers. Accelerated testing utilizing a combination of high stress, elevated temperature and a strong oxidizing agent has been used to determine the long-term 
performance of geomembranes [26]. Disinfectants (oxidizers) in potable water can severely degrade the inner surface of plastic pipelines, causing significant crack formation and eventually a complete failure. Service lifetimes of the failed water pipes vary between 1 to 25 years, which are significantly lower than the expected lifespan of 50 to 100 years [12]. Published data for these applications have confirmed that the interaction between stresses and environment could significantly accelerate the polymer degradation and lead to crack formation and propagation, referred to as stress corrosion cracking (SCC). Therefore, understanding the interaction between chemical degradation and fracture behavior for SCC is crucial for the design of polymer structural components for a target lifetime requirement.

Over the past few decades, both experimental and numerical studies have been performed to investigate creep crack growth (CCG) and SCC of polymers. Due to the long service time of polymer components, testing is usually accelerated by elevating the temperature and increasing reactant concentration (for SCC). Lu et al. [23] conducted extensive tests to study the failure time due to creep of a polyethylene copolymer. Specimens were tested at high temperatures (up to $80^{\circ} \mathrm{C}$ ) and different stress levels. Then, time-temperature superposition was used to extrapolate the lifetime-stress relation at room temperature from the higher temperature experiment results. Hsuan et al. [16] developed a Notched Constant Tensile Load test to evaluate the stress corrosion resistance of high density polyethylene geomembranes. In this test, single edge notched dogbone specimens are immersed in a highly corrosive environment (10\% Igepal) at an elevated temperature when constant tensile stresses are applied. The ultimate specimen failure time, as well as the applied stress, reveal the resistance of the tested resin to SCC.

The accelerated testing method is a valuable tool to measure the polymer performance under CCG and SCC, at least on a comparative basis. For instance, pipe manufacturers can use this approach to select the best performing material among multiple candidates. However, using this approach to predict the polymer behavior at low temperature/reactant concentration via extrapolation has raised some concerns. Lang et al. [21] pointed out that structural changes in a material due to temperature variations and thermal/chemical aging may compromise, even invalidate the predictions made by this approach. For example, chlorine solution has been used in accelerated aging treatment of polyethylene [9]. However, stability of the free chlorine components depends on the temperature. Devilliers et al. [10] pointed out that both hypochlorous acid $(\mathrm{HClO})$ and the hypochlorite $(\mathrm{OCl})$ ion become unstable when temperature is higher than $40^{\circ} \mathrm{C}$. Therefore, the degradation mechanism in a chlorinated environment at high temperature is different from that under 
room temperature.

In contrast to experimental investigation of SCC, numerical simulation provides a more flexible means for the prediction of polymer lifetime. Performance of different materials and part geometries over the entire lifetime can be evaluated using finite element analysis (FEA). This method allows for the generation of an optimal solution within the entire design space. In addition, the coupling effects of multi-physics (thermal, mechanical, chemical, etc.) can be readily evaluated, which is usually impractical if pursued experimentally. Therefore, numerical simulation is a promising method for the accurate predictions of polymer lifetime under SCC.

FEA has been utilized for the prediction of PE failure under CCG. Hutar et al. [18] developed a simulation approach to estimate the lifetime of pressurized HDPE pipe. A power law of crack growth kinetics was extracted from a compact tension specimen under static loading at $80^{\circ} \mathrm{C}$. This kinetics relationship was used to calculate the total failure time for a particular crack growth history. The stress intensity factor at various crack lengths was evaluated using the FEA calculations. However, this method cannot be easily implemented at lower temperatures due to the difficulties in determining the fracture kinetics.

In order to predict failure under SCC, a chemical kinetics simulation must be coupled with the mechanics model. Choi et al. [3] developed a numerical simulation scheme of SCC in a thermoplastic material based on the crack layer theory. In this study, SCC is considered a superposition of creep induced aging and chemical degradation. The loss of material toughness due to these two mechanisms were characterized through a set of phenomenological power laws. A generic kinetic parameter was proposed to represent the rate of chemical corrosion. Both SCC and mechanically driven crack growth were simulated. The parametric approach in this study effectively reveals the similarities and differences between SCC and CCG.

However, one of the features of SCC is that it strongly depends on the diffusion process, and the migration of substances is critical to the corrosion of a structure. Olden et al. [25] developed a numerical method to study the effects of hydrogen embrittlement (HE) on steel property. HE is a diffusion driven mechanism that significantly corrodes steel. Hydrogen slowly diffuses into the process zone around the crack tip and accumulates there, resulting in reduction of material strength and toughness. In this study, the diffusion of hydrogen was incorporated in the finite element simulation, and the extent of degradation was predicted as a function of hydrogen concentration. A cohesive zone model was utilized to characterize the nonlinear 
fracture property and its variation with hydrogen concentration.

The methods developed by Choi et al. and Olden et al. have shown promises in predicting the SCC of polymer structures. However, diffusion controlled degradation of polymers poses additional challenges that must be addressed. For polymers, disinfectants and oxygen from the environment diffuse into the structure and react with the material in an oxidation-reduction process. Antioxidants are commonly added to retard the reactions by neutralizing the oxidizers. To investigate SCC in a polymer, the oxidation reactions must be simulated in situ with the fracture analysis. In addition, the diffusion of multiple reacting substances, including both oxidizers and antioxidants, should be predicted. This motivates the development of a general computational model that takes into account the interactions between chemical reactions, diffusion, and mechanical deformation.

\section{Chemo-mechanical model for time-dependent crack growth}

The objective of this work is to develop a unified chemo-mechanical model to predict the time-dependent crack growth in a viscoplastic polymer, when exposed to an oxidizing environment. The polymer component is decomposed into two regions, and region specific material models are implemented. The crack region refers to the crack propagation path which is influenced by diffusion and chemical reactions throughout the component lifetime. Therefore, the fracture property is both time-dependent and location-dependent. The bulk region refers to the rest of the material where some degradation is expected, but that degradation is lumped into the crack region for modeling purposes. In this region, a corrosion-independent viscoplastic constitutive model is utilized. As a result, the material creep under a low stress level can be well captured. In this study, a chemomechanical cohesive zone model (CZM) will be used to characterize the loss of fracture property due to molecule chain scission. A chemical kinetics diffusion model is coupled with the CZM to predict the time-dependent degradation of polymer material properties and its consequence on the fracture behavior. 


\section{Viscoplastic constitutive model}

To analyze the structural behavior of PE, phenomenological constitutive models are often used to characterize the nonlinear stress-strain behavior. Despite significant differences in form, many existing models were developed based on the phenomenological uniaxial stress and strain relationship. Kurtz et al. ([19]) proposed an exponential function to describe the tensile true stress and strain behavior of irradiated and oxidized ultra-high molecular weight polyethylene. This is a rate-independent model in which true stress is a function of material density and strain. The model is applicable to the case where the strain is below 12\%. Liu et al. [22] developed nonlinear time-dependent models for PE based on the multi-Kelvin element theory and a power law function. The relevant model parameters can be determined by creep tests at various stress levels. The models are shown to yield good predictions at low stress rate $(0.01 \mathrm{MPa} / \mathrm{s})$ for small strains $(<0.015)$. Colak et al. [6] proposed an overstress model to characterize the viscoelastic and viscoplastic behavior of HDPE. Model parameters are determined from monotonic and cyclic uniaxial testing at different strain rates. Drozdov et al. [11] developed a viscoelastic-viscoplastic model for semi-crystalline polymers, and demonstrated its application in HDPE. Hamouda et al. [15] presented an elastic-viscoplastic model that contains two viscoplastic components, which account for the material behaviors at both high and low strain rates.

For creep loading, which is the focus of this study, a viscoplastic model is needed to capture the nonlinear strain history. Zhang et al. [29] proposed a viscoplastic model to characterize the rate-dependent behavior of PE. This model, which was developed on the framework of Bodner's theory [2], assumes that yielding occurs during all stages of loading. To account for the rate dependence and strain hardening effects, a hardening state variable $X$ was introduced to relate stress with plastic strain rate using a power-law function:

$$
\dot{\boldsymbol{\varepsilon}}^{p}=C\left(\frac{\sigma}{X}\right)^{n} \quad(n \geq 1)
$$

In this formula, $\sigma$ refers to the uniaxial stress, and $\dot{\varepsilon}^{p}$ denotes the uniaxial plastic strain rate. $C$ and $n$ are material constants. $X$ is a material variable that is a function of plastic strain work. This constitutive model was successfully employed to characterize the rate dependent behavior of HDPE in various loading conditions. In this study, we will adopt Zhang's model for the reasons states above. 


\section{Cohesive zone model}

The cohesive zone model has been widely used to predict the fracture in metal [25], composites [8], concrete [28] and polymers [13]. For example, Maiti et al. [24] developed a CZM to simulate the fatigue crack growth in epoxy based on the bilinear traction separation form. Elices et al. [13] simulated the compression/tensile testing of PMMA specimens via a combination of CZM and a nonlinear bulk constitutive model, and accurately predicted the load-displacement curves. In this study, a CZM with linear softening traction separation relation will be utilized, as shown in Fig.1. $\tau_{\max }$ is the maximum traction, which is representative of the material strength. $\delta_{c}$ is the separation displacement at failure. The fracture energy is denoted by $G_{I c}$.

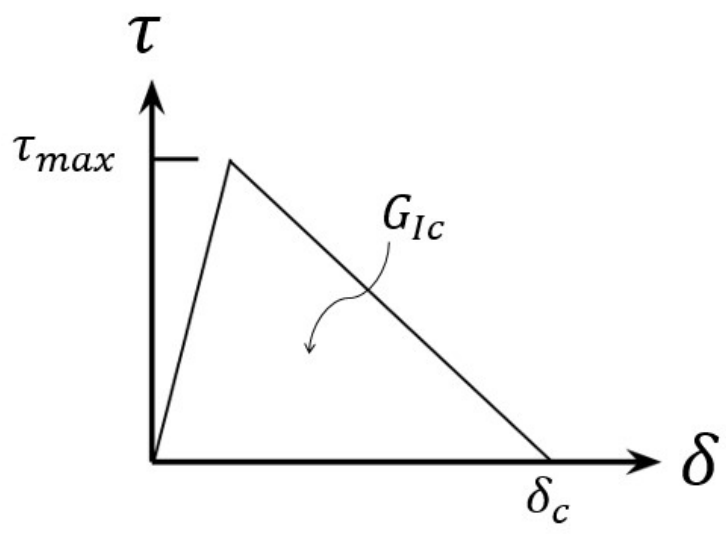

Figure 1: CZM with linear softening traction separation relation

\section{Chemical diffusion and reaction model}

In this study, we consider PE to be exposed to chlorine as an oxidizing agent. Such a corrosion environment is typical for PE pipes for water delivery. Antioxidants are added to PE during pipe fabrication to prevent the material from being corroded. Such antioxidants react with the oxidizing disinfectants and slowly get removed from the resin over time. Furthermore, this reaction process is accelerated by the migration of antioxidants into the surrounding liquid, as well as the penetration of disinfectants through the contact surface. After the antioxidants are depleted, PE will directly react with the disinfectants and oxygen, causing polymer chain scission and subsequently shorter molecular chains (and lower molecular weight). Consequently, both the strength and fracture energy of the material will be degraded.

In a recent study by Colin et al. [7], a comprehensive chemical kinetics and diffusion model was developed 
to predict the molecular weight loss of polyethylene due to reactions with chlorine dioxide (DOC). Through the simulation of chemical reactions between PE and various reactants, the molecular weight profile of PE can be determined through the pipe thickness over time. In general, the kinetics model incorporates the reactions between oxygen, Chlorine dioxide, antioxidant, and polyethylene. DOC can directly react with PE and produce active hydrocarbon radicals (see [7] for detailed descriptions of these chemical reactions). Reactions between these radicals result in the cross linking of hydrocarbon structures, thereby increasing the molecular chain length. On the other hand, radical oxidation reactions can also produce hydroperoxides, which decomposes and produces more hydrocarbon radicals results in molecular chain scission. The two opposite mechanisms, cross linking and chain scission, compete with each other and in conjunction determine the molecular weight of the polymer. The number of crosslinking $\bar{x}$ and chain scission $\bar{s}$ can be related with the molecular weight by:

$$
\frac{1}{M_{w}}-\frac{1}{M_{w}^{0}}=\frac{\bar{s}}{2}-2 \bar{x}
$$

where $M_{w}^{0}$ is the weight average molecular weight in the virgin state, and $M_{w}$ is the molecular weight after reactions initiate. The reaction and diffusion of substances are modeled in a fundamental mathematical form based on Fick's law [1]:

$$
\frac{\partial[C]}{\partial t}=D \frac{\partial^{2}[C]}{\partial x^{2}}-r([C])
$$

where, $[C]$ denotes the concentration of a reactant $C$, and $x$ represents the depth that $C$ penetrates. The rate of reaction depletion $r$ is a function of the reactant concentration. $D$ represents the coefficient of diffusion, which is dependent on temperature. Therefore, running the chemical kinetics model involves solving a set of differential equations that eventually generates the molecular weight profile in a 1D space (that is, along the direction of diffusion). It should be noted that all the kinetic parameters (rate constant $k$ and coefficient of diffusion $D$ ) are a function of temperature, and the temperature dependence can be calculated using the Arrhenius equation. 


\section{Molecular weight dependence of CZM parameters}

For a semicrystalline polymer like PE, both the crystalline and amorphous states contribute to the material strength. Under room temperature, which is well above the material glass transition temperature $T_{g}$, the amorphous region is liquid-like and the Van der Waals bonding is close to zero. Therefore, the load carrying capacity is primarily attributed to the crystalline regions, which are joined together via tie-molecules. Huang et al. [17] proposed that under room temperature, the strength of semicrystalline polyethylene is proportional to the number of tie-molecules, which is a linear function of weight average molecular weight $M_{w}$. In addition, a threshold molecular weight $M_{c}$ was identified, below which no tie molecules can form. Hence, based on the molecular structure of polyethylene, the material strength can be considered proportional to $M_{w}$. In the context of CZM, $\tau_{\max }$ is representative of the strength. Therefore, the following relation is proposed:

$$
\tau_{\max } \propto\left(M_{w}-M_{c}\right)
$$

In the framework of continuum damage mechanics, damage extent is described by a scalar parameter $\omega$, which is usually determined by the material strains. In this study, we adopt the similar concept, and define the damage parameter $\omega$ as a function of molecular weight:

$$
\omega=1-\left\langle M_{w}-M_{c}\right\rangle /\left(M_{w}^{0}-M_{c}\right)
$$

where $\langle x\rangle=\max (x, 0), M_{w}$ is the molecular weight of the degraded state, and $M_{w}^{0}$ represents the virgin state. Accordingly, the peak traction $\tau_{\max }$ can also be defined as a function of $\omega$ :

$$
\tau_{\max }=\tau_{\max }^{0}(1-\omega)
$$

Similarly, $\tau_{\max }^{0}$ is the strength of the undegraded material. The loss of molecular weight also results in a rapid reduction of toughness. A recent study by Choi et al. suggests a power law relationship between the fracture energy and damage parameter [4]:

$$
G_{I c}=G_{I c}^{0}(1-\omega)^{m}
$$


where $G_{I c}^{0}$ is the toughness of virgin material. For PE, the constant $m$ was found to be 2.4, and is adopted in this study. It should be noted that, with such a $m$ value, the Irwin's characteristic length, $l_{c h}=E G_{I c} / \tau_{\max }^{2}$, decreases as $\omega$ increases, which indicates that the degraded material becomes more brittle.

\section{Determination of model parameters}

The parameters of each component of the proposed model are determined by separate tests. In a recent study by the authors [14], tensile testing of dogbone specimens was performed at various strain rates to find the viscoplastic model constants. The CZM parameters of the undegraded HDPE are calibrated with the fracture tests done by Kwon et al. [20]. The chemical kinetics and diffusion coefficients are calculated based on the constants provided by Colin et al.[7].

\subsection{Viscoplastic model parameters}

The bulk material model parameters were determined through uniaxial tensile testing at various strain rates under room temperature $\left(20^{\circ} \mathrm{C}\right)$. The true stress and strain relationships at each strain rate were extracted with the aid of video imaging. Details of this experimental procedure can be found in [14]. In the current study, the material used is a high density polyethylene (HDPE) sheet acquired from McMaster-Carr. Micro tensile testing specimens (as specified by ASTM D-1708) were prepared via die-cutting of 1/16" thick sheets. Three levels of strain rates were considered during tensile testing: $1.36 \times 10^{-4} / \mathrm{s}, 1.36 \times 10^{-3} / \mathrm{s}$ and $1.36 \times 10^{-2} / s$. After the true stress and strain relations were obtained, the experimental data was fitted to the bulk constitutive model by least square optimization method.

Fig. 2 presents the true stress and strain relations from both experimental measurements and numerical prediction. In this study, the upper strain limit in tensile testing is $15 \%$. Thus, the bulk constitutive model is calibrated up to this strain limit. This bulk strain threshold will be taken as a material failure criterion during finite element simulation. The bulk constitutive model parameters, as obtained from material calibration, are listed in Table1: 


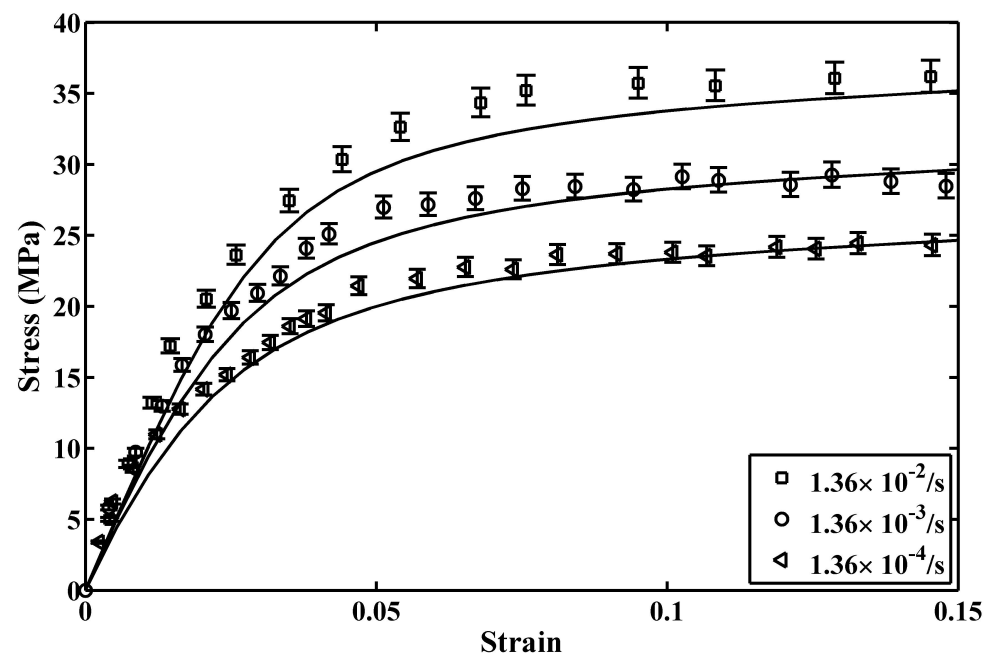

Figure 2: Stress and strain relations of polyethylene at various rates [14]

Table 1: Bulk constitutive model parameters at $20^{\circ} \mathrm{C}$

\begin{tabular}{cccccccccc}
\hline$E$ & $C$ & $n$ & $\beta$ & $\gamma$ & $d_{1}$ & $d_{2}$ & $d_{3}$ & $X_{0}$ & $v$ \\
\hline $1150 \mathrm{MPa}$ & 0.01 & 8 & $4 \times 10^{-5} \mathrm{MPa}$ & $1 \times 10^{-4} \mathrm{MPa}$ & $9.67 \times 10^{-4}$ & 3.59 & 0.019 & $1 \mathrm{MPa}$ & 0.38
\end{tabular}

\subsection{Cohesive zone model parameters for undegraded HDPE}

For a cohesive zone model with a bilinear traction separation relationship, we need to determine the cohesive strength and fracture energy of the undegraded material. The parameter calibration was performed based on tensile test data of double edge notched (DEN) HDPE specimens reported in the literature [20]. The specimens were $12.5 \mathrm{~mm}$ thick commercially extruded HDPE plates acquired from McMaster Carr. This material is identical with the material used to extract the stress-strain relations. The ligament lengths varied between $4 \mathrm{~mm}$ and $12 \mathrm{~mm}$.

Typically, researchers utilize thin DEN polymer specimens in fracture experiments to extract the fracture toughness under the plane-stress state, which is significantly greater than the plane strain toughness for polyethylene. The ultra high ductility of PE enables the specimen to undergo very large out-of-plane deformation when the thickness is small with respect to the ligament length. By contrast, when the thicknesses are greater than the ligament length, the specimens approach the plane strain state, and the failure mode is brittle [20]. This type of fracture is similar to SCC of polymer pipes, which occurs in plane strain conditions. For thickness to ligament length ratios greater than $1(T / L>1)$, the stress state approaches the plane strain 
condition. For the special case of pipes, $T$ corresponds to the total pipe length, while $L$ is equal to the pipe wall thickness. Thus, the DEN specimens utilized in fracture parameter calibration [20], where $T / L>1$, are acceptable for the simulation of crack growth in pipes.

It is clear that $\tau_{\max }$ denotes the material strength prior to damage initiation. In a constant crosshead speed tensile test, the applied load increases monotonically until the peak is reached. Thereafter, the crack grows through the entire ligament, accompanied by damage propagation. The nominal section strength is defined as normalized maximum load $\left(F_{\max }\right)$ with respect to ligament area: $\sigma_{\text {nom }}=F_{\max } /(T \cdot L)$. Accordingly, $\tau_{\max }$ can be found at the instant of peak load. However, the magnitude of $\sigma_{\text {nom }}$ varies with ligament length. Based on the tensile testing data of DEN samples in [20], $\sigma_{\text {nom }}$ increases monotonically with decreasing ligament length as shown in Fig.3. Hence, $\tau_{\max }$ for plane strain condition can be obtained by extrapolating $\sigma_{\text {nom }}$ to $L \rightarrow 0$, in which case the entire ligament would have a constant stress profile. Therefore, $\tau_{\max }$ is equal to $\sigma_{\text {nom }}$ for $L \rightarrow 0$. In our study, $\tau_{\max }^{0}=40 \mathrm{MPa}$ will be utilized for the strength of virgin material.

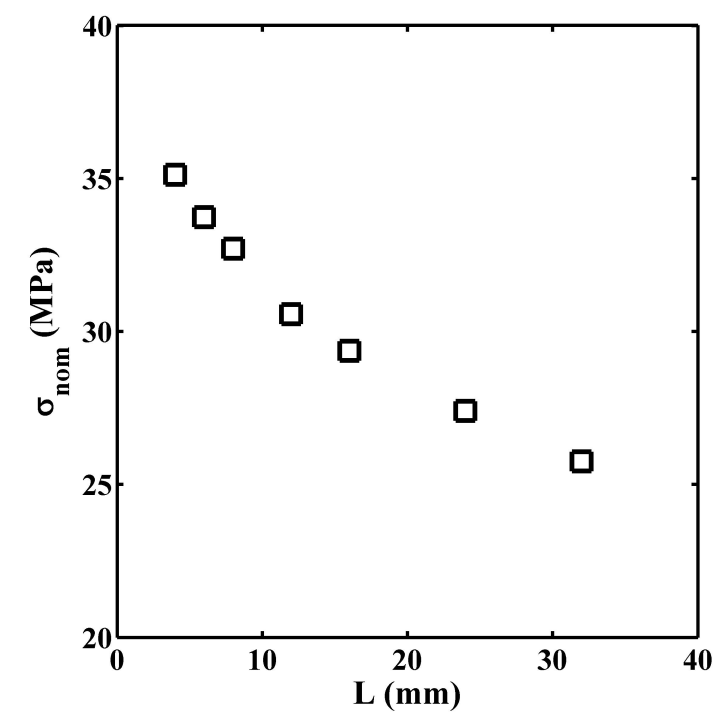

Figure 3: Nominal section strength as a function of $\mathrm{L}$

After $\tau_{\text {max }}^{0}$ is determined, the fracture energy can be determined by matching the predicted peak load with experiments. Using data from [20] for the $4 \mathrm{~mm}$ ligament specimen, $G_{I c}^{0}$ is curve fit to be $12 \mathrm{MPa} \cdot \mathrm{mm}$. Due to the great variety of data extrapolation approaches, plane strain fracture toughness varies from below $6 \mathrm{MPa} \cdot \mathrm{mm}$ [20], to greater than $20 \mathrm{MPa} \cdot \mathrm{mm}$ [27]. Hence, $12 \mathrm{MPa} \cdot \mathrm{mm}$ is a reasonable fracture toughness value for numerical simulation. 


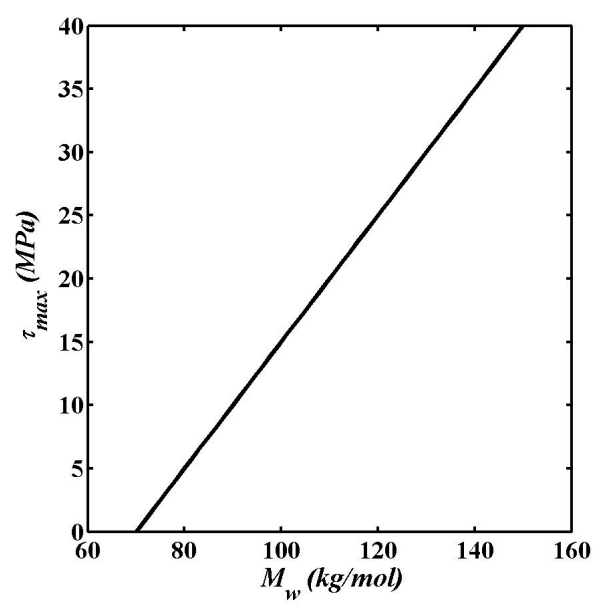

(a) $\tau_{\max }$ vs. $M_{w}$

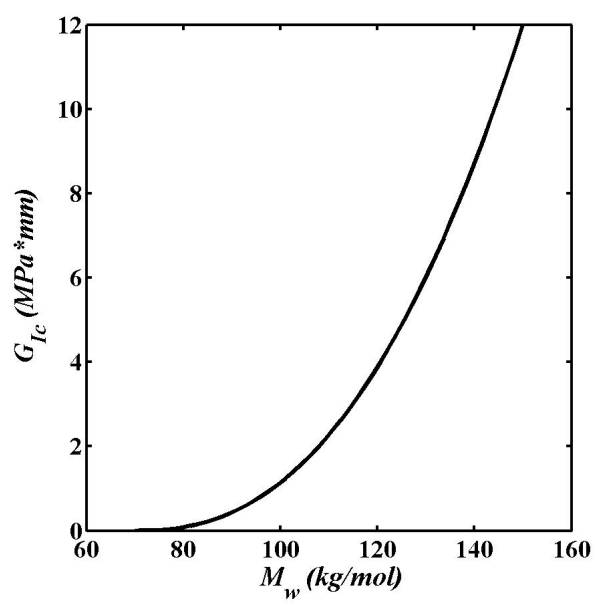

(b) $G_{I c}$ vs. $M_{w}$

Figure 4: Molecular weight dependence of $\tau_{\max }$ and $G_{I c}$

\subsection{Chemical reaction and diffusion constants}

To successfully simulate the mechanical property loss due to chain scission of HDPE, the threshold value of the molecular weight $M_{c}$ needs to be determined. Colin et al. pointed out that when the weight average molecular weight of PE drops below $70 \mathrm{~kg} / \mathrm{mol}$, cracks initiate and propagate easily, regardless of its initial molecular weight [7]. Therefore, $M_{c}=70 \mathrm{~kg} / \mathrm{mol}$ is used in the numerical simulation. Given the assumption of $M_{c}$, the cohesive parameters $\tau_{\max }$ and $G_{I c}$ can be calculated as a function of $M_{w}$. The results are shown in Fig.4. Here, an initial molecular weight of $150 \mathrm{~kg} / \mathrm{mol}$ is considered for the undegraded HDPE. Note that $\tau_{\max }^{0}=40 \mathrm{MPa}$ and $G_{I c}^{0}=12 \mathrm{MPa} \cdot \mathrm{mm}$ are used for strength and toughness.

The chemical kinetics diffusion modeling approach has been discussed in Section 2. For our study, kinetics simulations have been performed for $20^{\circ} \mathrm{C}$. Accordingly, all the kinetics parameters (including rate constants and diffusion coefficients) have been calculated for this temperature based on an Arrhenius law.

\section{Numerical implementation and case study}

In this paper, two types of specimen geometries and loading conditions will be simulated using FEA. Schematic drawings of the two cases are shown in Fig.5. Monotonic loading of double edge notched (DEN) specimens (see Fig 5a) is simulated to replicate the fracture tests done by Kwon et al. [20]. A constant 


\begin{tabular}{cc|cc}
\hline Rate constant & Value at $20^{\circ} \mathrm{C}$ & Rate constant & Value at $20^{\circ} \mathrm{C}$ \\
\hline$k_{1 u}$ & $9.05 \times 10^{-13}$ & $k_{6 a}$ & $2.72 \times 10^{5}$ \\
$k_{1 b}$ & $5.46 \times 10^{-10}$ & $k_{6 b}$ & $2 \times 10^{6}$ \\
$k_{1 d}$ & $2.7 \times 10^{-5}$ & $k_{6 c}$ & $1.54 \times 10^{5}$ \\
$k_{2}$ & $10^{8}$ & $k_{6 d}$ & $3.81 \times 10^{6}$ \\
$k_{3}$ & $1.47 \times 10^{-3}$ & $k_{7}$ & $1.15 \times 10^{6}$ \\
$k_{4}$ & $8 \times 10^{11}$ & $k_{1 s}$ & $5 \times 10^{-2}$ \\
$k_{5}$ & $1.33 \times 10^{11}$ & $k_{s 1}$ & 1.67 \\
\hline
\end{tabular}

Table 2: Rate constants at $20^{\circ} \mathrm{C}$ for kinetic modeling. Unit $=L /(\mathrm{mol} \times s)$

\begin{tabular}{cc}
\hline Coefficient of diffusion & Value at $20^{\circ} \mathrm{C}$ \\
\hline$D_{O 2}$ & $2.49 \times 10^{-11}$ \\
$D_{D O C}$ & $2 \times 10^{-11}$ \\
$D_{A O}$ & $2.2 \times 10^{-16}$
\end{tabular}

Table 3: Coefficients of diffusion at $20^{\circ} \mathrm{C}$ for kinetic modeling. Unit $=\mathrm{m}^{2} / \mathrm{s}$

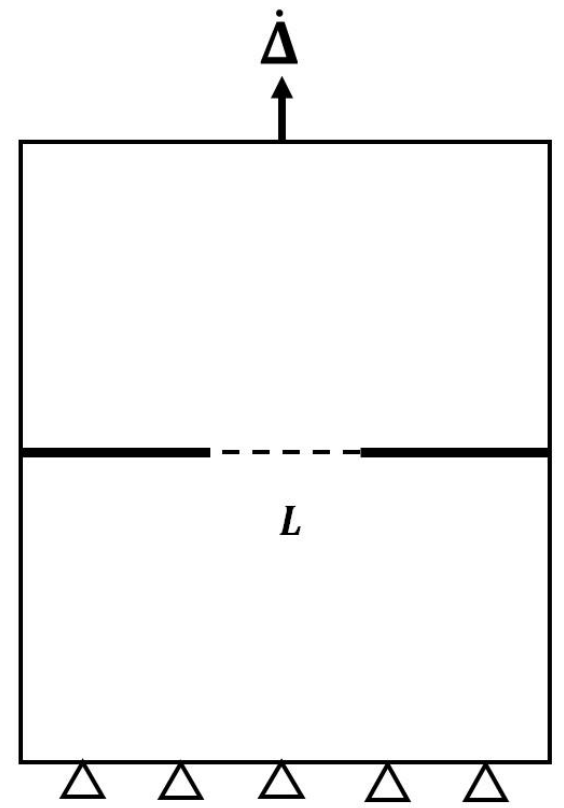

(a) DEN specimen

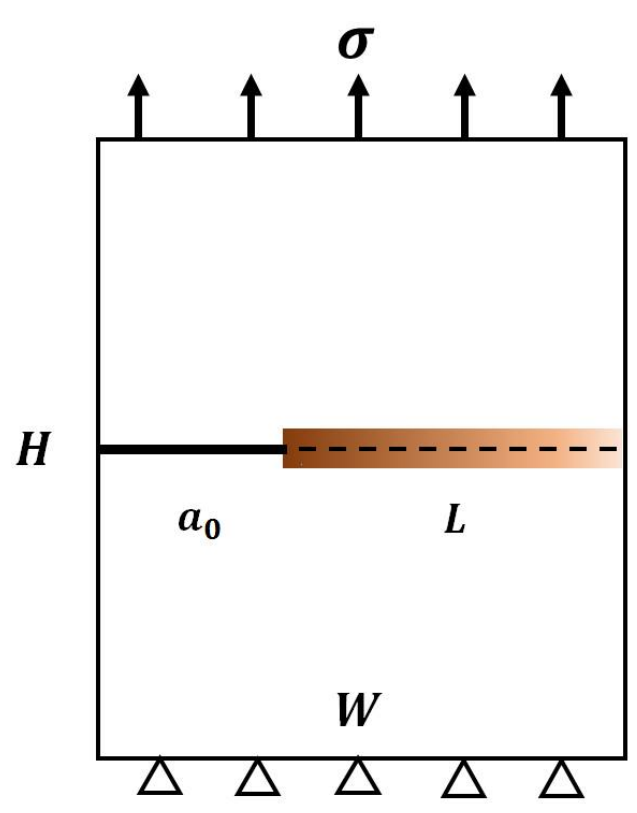

(b) SEN specimen

Figure 5: DEN and SEN specimens in finite element analysis 
displacement rate $\dot{\Delta}$ is prescribed on the upper specimen edge. To study the SCC, a single edge notched (SEN) specimen (Fig 5b) is utilized to represent an HDPE component with an initial crack. In this case, a constant load $\sigma$ is applied on the upper edge. The bold lines indicate pre-cracks, and the dashed lines represent the ligaments. For these two specimens, we expect that mode-I fracture will take place along the ligament. Therefore, we place the CZM along the ligament as the potential crack growth path.

\subsection{Simulation of Stress Corrosion Cracking}

\subsubsection{Numerical implementation of the proposed model}

In this study, we simulate the SCC of HDPE in a chlorinated environment. The polymer component, after being exposed to chlorine, undergoes continuous chemical corrosion assisted by the diffusion of oxygen, chlorine dioxide and antioxidant. As the surface material becomes severely damaged, fracture takes place and the crack front inches forward. Meanwhile, the region around crack tip that is subject to chemical reactions and diffusion also moves over the time. Therefore, the simulation needs to take into account the coupling of the following mechanisms: 1) creep damage under a constant stress; 2) reactions of oxidizers (chlorine dioxide and oxygen) with antioxidant and polyethylene, and 3) diffusion of oxidizers and antioxidant.

Fig.5b shows a single edge notched HDPE specimen subjected to a corrosive environment. The left edge of the specimen is in contact with the corrosive environment, and will be referred to as the inner surface. The right edge is in contact with air, and represents the outer surface. The pre-crack represents a defect on the inner surface. The region that is affected by chemical diffusion and reactions is superimposed on the ligament. It should be stressed that the structural performance is only determined by the degradation along the ligament under mode I fracture, despite that a much larger area proximate to the crack surfaces is affected by diffusion. As the crack grows along the ligament, the degraded region will expand toward the outer surface. To capture the influence of degradation, chemical kinetics simulation must be performed along the entire ligament, and the boundary condition should be updated continuously with crack propagation.

Procedure to implement the coupled mechanical-chemical simulation is described as follows. We first start the chemical kinetics and diffusion simulations by prescribing the initial condition (IC) and boundary condition (BC). This involves the assignment of the concentration of various reactants through the entire 
ligament. The kinetics simulation is performed in Matlab, to obtain the molecular weight profile along the crack growth path as a function of both location and time: $M_{w}(x, t)$. Next, the $M_{w}$ profile is mapped to all the cohesive elements in Abaqus via a user defined subroutine UFIELD. Subsequently, finite element analysis is performed, with $\tau_{\max }$ and $G_{I c}$ calculated for each cohesive element in every time increment based on the $M_{w}$ assignment. The status of the cohesive element at the crack tip is monitored continuously, and the finite element simulation is paused when this cohesive element is detected failure. This indicates that the crack will advance by the length of one cohesive element ( $0.1 \mathrm{~mm}$ in this study).

When the crack progresses during the simulation, the IC and BC are updated accordingly in the kinetics simulation. Then, the kinetic simulation must be repeated with the changed IC and BC. In general, the crack growth is simulated through such an iterative process until a global failure criterion is satisfied, indicating ultimate damage of the specimen. In this study, the specimen is considered to fail either when the maximum principal strain in the bulk region reaches $15 \%$ or when unstable crack growth occurs.

\subsubsection{Finite element analysis implementation}

A comprehensive co-simulation approach incorporating both finite element analysis (in Abaqus 6.13) and chemical kinetics/diffusion simulation (in Matlab 2010) was developed. In the FE modeling, an elasticviscoplastic constitutive model is utilized to predict the creep behavior of polyethylene, implemented through a user defined material (UMAT) subroutine. The cohesive zone model is applied to capture the nonlinear fracture property. To account for the influence of the oxidizing environment, a diffusion controlled oxidation reaction simulation is carried out to compute the molecular weight degradation of PE, so as to estimate the loss of fracture toughness. The chemical diffusion reaction calculations are performed in Matlab, and the results are integrated with Abaqus via a user defined subroutine .

The dimensions of the SEN specimen are $7.3 \mathrm{~mm} \times 4.5 \mathrm{~mm}(H \times W)$, with an initial crack length $a_{0}=$ $0.3 \mathrm{~mm}$. The specimen bottom is fixed in all degrees of freedom, and a constant traction $\sigma$ is applied on the upper surface. Cohesive elements are placed along the crack propagation path $L$. The bulk region is modeled by $3 \mathrm{D}$ solid elements, and the elastic-viscoplastic constitutive model is implemented. Cohesive elements are placed in the cohesive zone, and the nonlinear traction separation relation is applied.

The simulation of crack propagation under stress corrosion is performed for room temperature. Three 


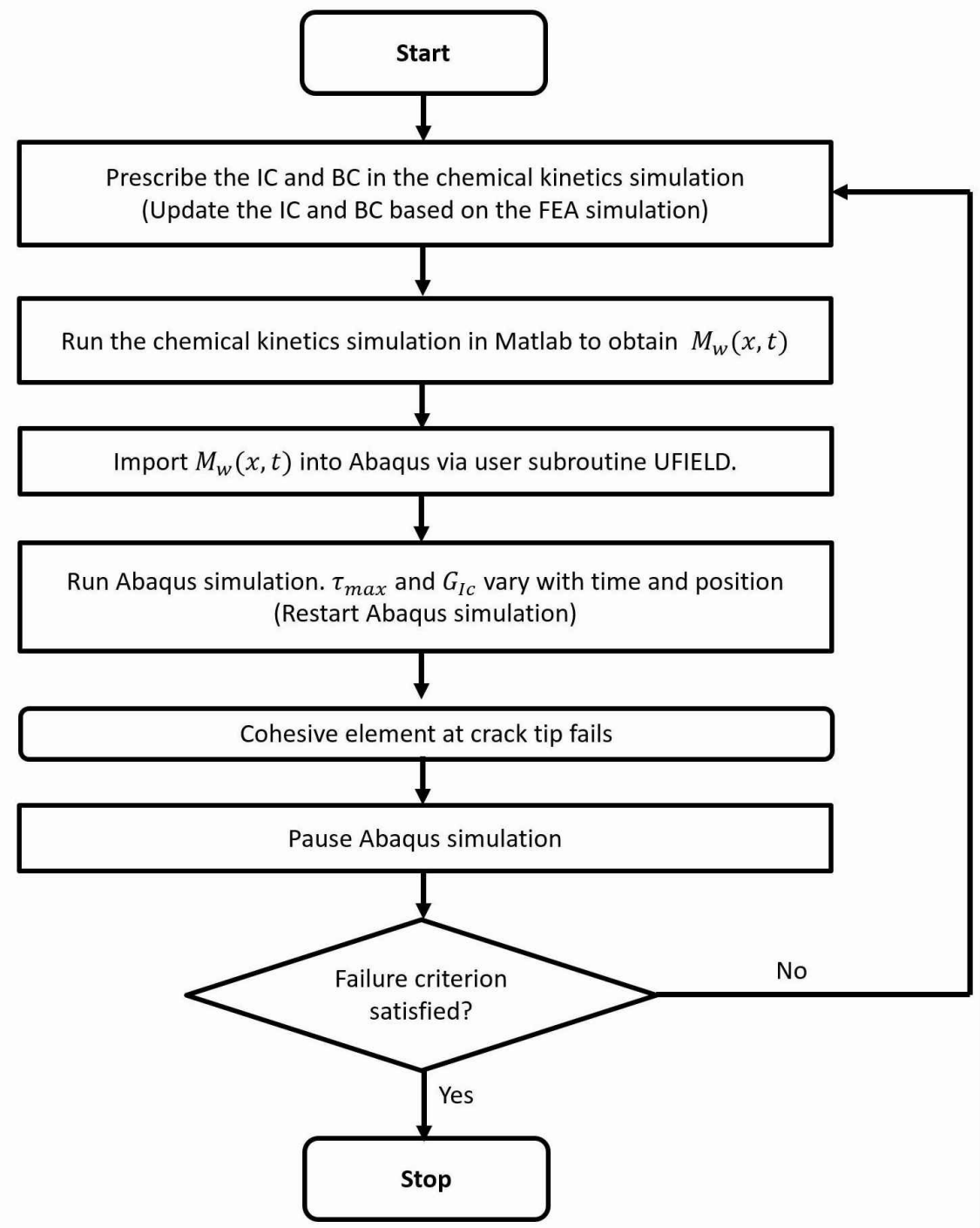

Figure 6: Algorithm of implementing coupled simulation of mechanics and chemical diffusion reaction 
levels of chlorine dioxide concentration were investigated: $0.0005 \mathrm{ppm}, 0.01 \mathrm{ppm}$, and $0.15 \mathrm{ppm}$, representing increasing rates of degradation. For each level of oxidizer concentration, the simulation was conducted for various stress levels. The crack growth history and the ultimate lifetime of the specimen were obtained in each scenario.

\section{Results and discussion}

In this section, the results from two sets of simulation are presented and discussed. First, the fracture testing of undegraded DEN specimens is predicted and compared with the experimental data. Next, the two failure mechanisms of HDPE under SCC are explored and discussed.

\subsection{Undegraded DEN specimens under monotonic loading}

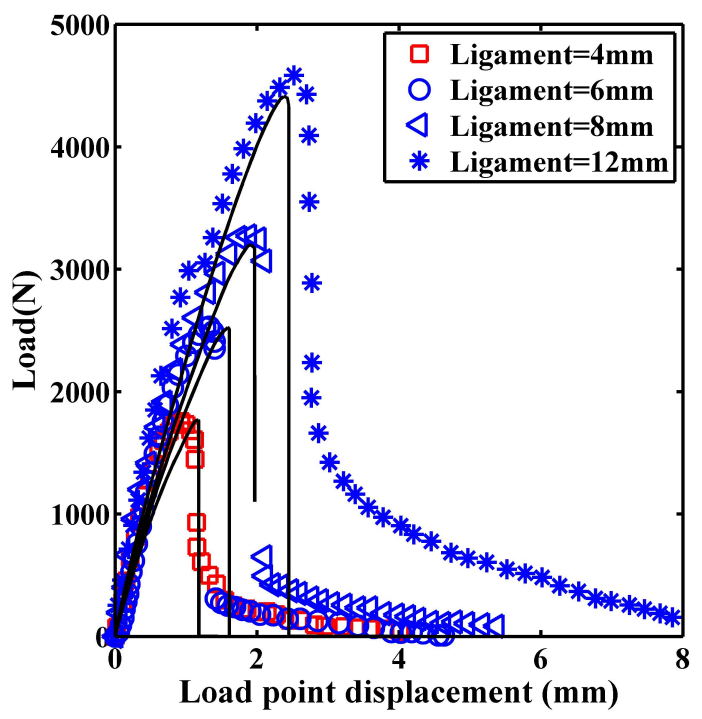

Figure 7: Load displacement curves for specimens of various ligament lengths. Ligament=4mm is used to calibrate $G_{I c}^{0}$

With the determined cohesive zone model parameters, fracture tests on DEN specimens provided by Kwon et al. [20] were simulated. Fig.7 shows the load displacement curves for 4 specimens (with various initial ligament lengths) from the published experimental data and the simulation results. The peak load in each tensile test has been accurately predicted. Note that for each experiment, the applied load dissipates 
dramatically after peak (regime 1), followed by a more gradual softening (regime 2). The mechanisms of the two regimes in load softening were revealed by Kwon et al. [20]. In regime 1, rapid load drop occurs due to brittle fracture at the center of specimen thickness. At the same time, fibrils on the specimen surface are still connected, and undergo large plastic deformation with increasing displacement. This corresponds to the gradual post-peak load drop (regime 2). In fact, the extent of surface necking is reduced with larger $T / L$ due to the dominance of the plane strain condition. Hence, it can be expected that for a perfect plane strain condition, failure caused by plastic flow will be negligible compared with brittle fracture.

It should be mentioned that regime 2 in the load softening can also be accurately captured if a more sophisticated CZM softening law (such as bilinear softening) were selected. However, the failure mode in regime 2 is not important in the simulation of brittle fracture under SCC. Therefore, the current modeling scheme, which relies on parameters found from plane strain data, is appropriate for predicting crack growth in polymeric pipes.

\subsection{SEN specimens under sustained loading in a chlorinated environment}

In addition to the DEN specimen under monotonic loading, we also simulate the crack growth in SEN specimen exposed to a chlorinated environment. The crack length history can be extracted from each SCC simulation. Fig. 8 presents the calculated time evolution of the crack length for three [DOC] concentration levels, all at a stress level of $1.11 \mathrm{MPa}$, which is a typical stress value for a municipal water transportation system [7]. In general, the crack initiates earlier at higher [DOC] concentration due to faster chemical degradation and subsequently loss of toughness. Two distinct patterns of crack propagation can be identified. For $[D O C]=0.15 \mathrm{ppm}$ and $0.01 \mathrm{ppm}$, the crack growth rate is almost constant until the strain criterion $\left(\varepsilon_{\max }=0.15\right)$ is reached. In contrast, for $[\mathrm{DOC}]=0.5 \times 10^{-4} \mathrm{ppm}$, the crack grows at a constant rate up to $a=2 \mathrm{~mm}$, as indicated by point A in Fig.8. Thereafter, the fracture accelerates until ultimate failure. In the following discussion, $[\mathrm{DOC}]=0.15 \mathrm{ppm}$ and $0.01 \mathrm{ppm}$ will be referred to as the high concentration case,

and $[D O C]=0.5 \times 10^{-4} \mathrm{ppm}$ is the low concentration case. The simulated results can be explained by the complex interaction between chemical reactions, diffusion, and material creep. Due to the differences in disinfectant concentration, either chemical reaction or diffusion would play a dominant role, which affects the crack growth behavior. To understand the effect of concentration on the SCC behavior, we will investigate the simulated crack growth rate and polymer degradation extent along the ligament. 


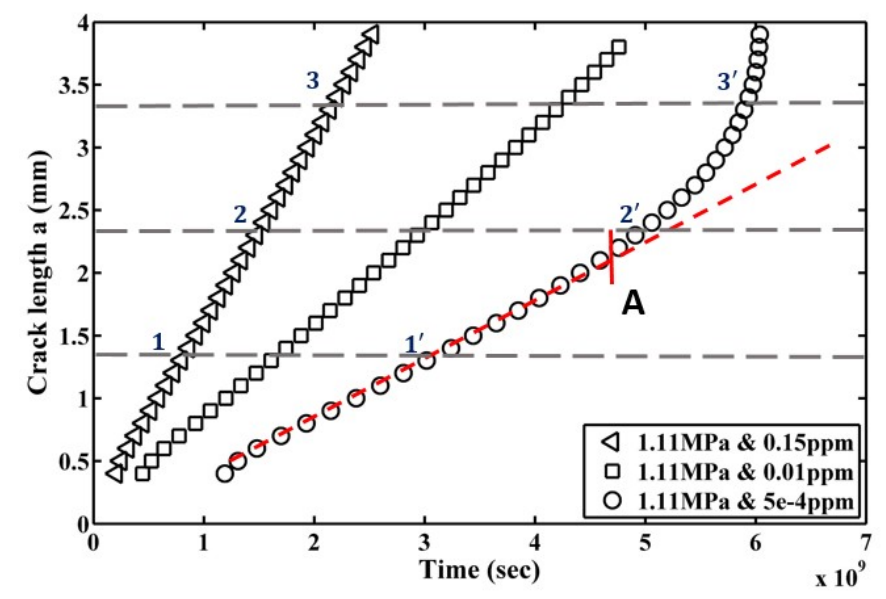

Figure 8: Crack growth history when stress=1.11MPa

The central difference method was used to compute the crack growth rate $(d a / d t)$ for each scenario. The results are presented as a function of stress intensity factor $K$ at the crack tip. For the high concentration environment (Fig.9a), $d a / d t$ is almost a constant for both concentrations, regardless of the stress intensity factor $K$. This indicates that crack growth is governed by the chemical reactions and independent of applied stress. By contrast, in the low concentration case (Fig.9b), $d a / d t$ monotonically increases by almost two orders of magnitude before reaching the strain limit. In this case, crack propagation is stress driven. The other observation is that in the high concentration (eg. $[\mathrm{DOC}]=0.15 \mathrm{ppm})$, the $d a / d t$ vs $K$ curves at various stresses overlap, while at low concentration no overlapping is observed.

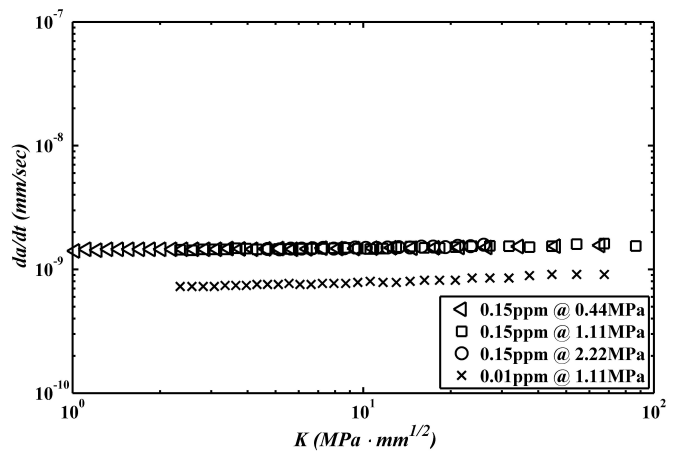

(a) High concentration set

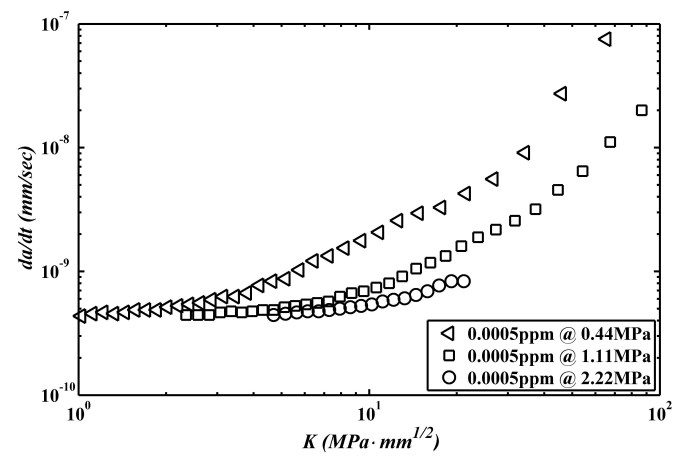

(b) Low concentration set

Figure 9: Crack growth rate versus stress intensity factor for two distinct sets

Based on the proposed model, it is clear that the evolution of the molecular weight $\left(M_{w}\right)$ profile would have a significant influence on the crack growth behavior. Fig.10 presents the $M_{w}$ profile through the remaining 


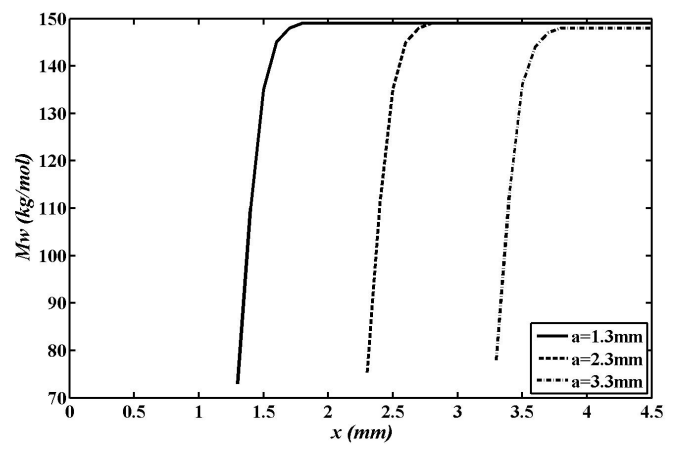

(a) $0.15 \mathrm{ppm}$

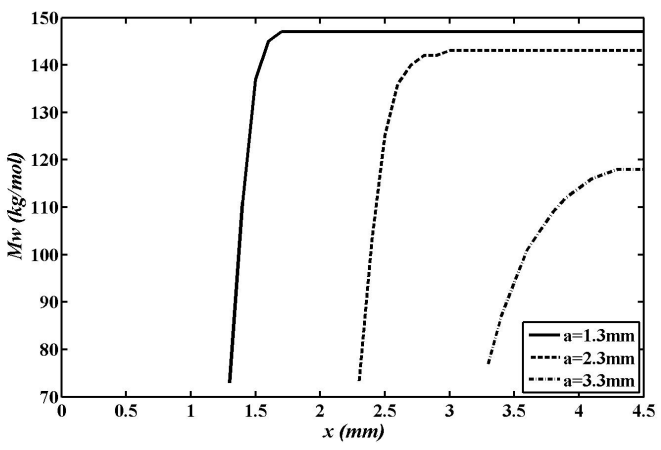

(b) $0.0005 \mathrm{ppm}$

Figure 10: Molecular weight profile for $0.15 \mathrm{ppm}$ and $0.0005 \mathrm{ppm}$, at three crack lengths respectively

ligament when [DOC] is $0.15 \mathrm{ppm}$ and $0.0005 \mathrm{ppm}$, both at a stress level of $1.11 \mathrm{MPa}$. In each scenario, the $M_{w}$ profile is presented at three crack lengths $(1.3 \mathrm{~mm}, 2.3 \mathrm{~mm}$, and $3.3 \mathrm{~mm}$, indicated by dashed lines in Fig.8). Note that the crack advances to a particular length at different times. For example, $a=1.3 \mathrm{~mm}$ at either $0.8 \times 10^{9} \mathrm{sec}$ for [DOC] $=0.15 \mathrm{ppm}(\mathbf{1})$, or $3.02 \times 10^{9} \mathrm{sec}$ for $0.0005 \mathrm{ppm}(\mathbf{1})$. The inner surface, where the edge of the specimen contacts the corrosive environment, is indicated by $x=0 \mathrm{~mm}$. The outer surface, which is in contact with air, is $x=4.5 \mathrm{~mm}$. It can be seen in both Fig.10a and Fig.10b, the $M_{w}$ profiles exhibit a steep molecular weight drop forms at the crack tip due to chemical reactions. However, the $M_{w}$ profiles far from crack tip differ significantly between the low and high concentration cases. In the high concentration environment, the $M_{w}$ (and consequently the mechanical properties) is at the initial value for most of the ligament. The degradation of properties only occurs in a small region of a size approximately equal to 0.5 $\mathrm{mm}$ at the crack tip. In the low concentration environment, the $M_{w}$ is severely reduced throughout the entire ligament as the crack tip advances.

The difference in $M_{w}$ profiles at high or low disinfectant concentration is caused by the relative dominance between the chemical reaction and diffusion processes. For the high concentration scenario, chemical reactions proceed at a fast rate. As a result, the oxidizers are quickly depleted before they could migrate far into the component, and the $M_{w}$ loss is localized near the surface. By constrast, reactions are slower at the low disinfectant concentration, and the oxidizers can diffuse farther into the remaining ligament. Therefore, as the disinfectant concentration drops, the polymer degradation mode transitions from reaction-dominant to diffusion-dominant.

The differences in the crack growth rate history (See Fig.9) can then be explained by the two polymer 


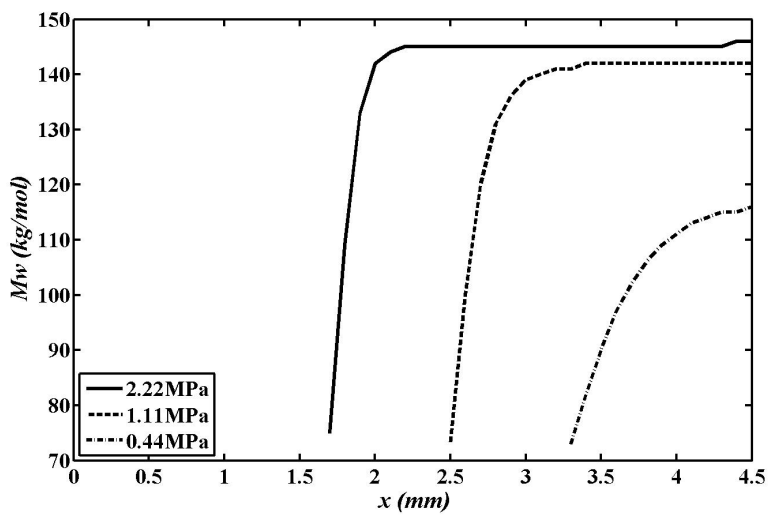

Figure 11: $M_{w}$ profiles for $[\mathrm{DOC}]=0.0005 \mathrm{ppm}$ at $\mathrm{K}=10 \mathrm{MPa} \cdot \sqrt{\mathrm{mm}}$

degradation modes. For reaction-dominant crack growth, $d a / d t$ is controlled by the concentration of the disinfectant concentration. Therefore, $d a / d t$ is constant when the disinfectant concentration does not change (Fig.9a). For diffusion-dominant crack growth, the monotonic increase of $d a / d t$ is predominantly influenced by the reduction of material property in the remaining ligament. In other words, crack growth becomes stress driven due to weakened material properties.

As also noted previously, the $d a / d t--K$ curves for the low concentration do not overlap with each other for different applied stress levels. In fact, for a given $K$, crack growth rate at a lower stress level is faster. This phenomenon can also be explained by reaction and diffusion mechanisms. Fig. 11 shows the $M_{w}$ profile for the three stress levels at the instant $K$ reaches $10 \mathrm{MPa} \cdot \sqrt{\mathrm{mm}}$ (see the dashed line in Fig.9b). Note that the same stress intensity factor corresponds to various crack lengths due to differences in stress level. At the lowest stress level (0.44MPa), the remaining ligament is most degraded because of long exposure time. In this case, the reduction of material property outweighs the influence of stress such that the crack grows fastest at this given $K$.

In addition to the crack growth history, we also investigate the ultimate failure times. For the two levels of [DOC] $(0.15 \mathrm{ppm}$ and $0.0005 \mathrm{ppm})$, simulation has been performed at various stress levels ranging from $0.44 \mathrm{MPa}$ to $8.9 \mathrm{MPa}$. All the scenarios simulated occur at $20^{\circ} \mathrm{C}$, consistent with the temperature that bulk material property was obtained.

Observation of pipe failure under SCC shows the failure mode depends on the level of applied stress ([7], [18], [5]). At high stresses, failure occurs in the form of localized bulging as a result of excessively large deformation. At low stresses, brittle failure takes place due to slow crack growth assisted by significant 


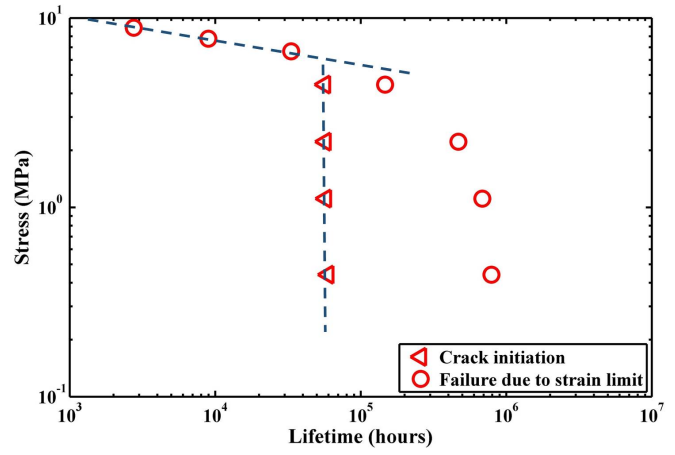

(a) $0.15 \mathrm{ppm}$

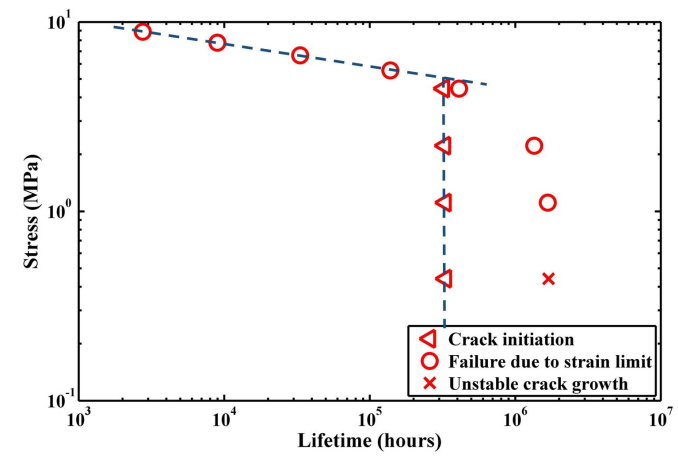

(b) $0.0005 \mathrm{ppm}$

Figure 12: Simulated lifetime-stress relations at $20^{\circ} \mathrm{C}$

chemical degradation. In the simulation results, both of these failure mechanisms are revealed. Fig.12b and 12a present the specimen lifetimes versus stresses, both on a log scale. At high stresses, the specimen undergoes significant deformation and reaches the bulk material strain limit. This occurs when the stress is greater than $5.5 \mathrm{MPa}$ at $[\mathrm{DOC}]=0.0005 \mathrm{ppm}$, or $6.6 \mathrm{MPa}$ at $[\mathrm{DOC}]=0.15 \mathrm{ppm}$. On the lifetime plot, an asymptotic line is formed in this regime. This trend line has a small slope, indicating relatively significant stress dependence. At low stresses, crack initiates (as shown by $\triangleleft$ ) and grows until failure either from large strain or unstable crack growth. Here, crack initiation is defined as the failure of the first cohesive element at crack tip (zero cohesive stress). It is noteworthy that for either DOC concentration, crack initiation time is almost constant, regardless of the applied stress. This is a clear indication that crack initiation is predominantly driven by the corrosion of $\mathrm{PE}$, and the effect of stress is insignificant. Additionally, crack initiation occurs at an earlier time for the high concentration as expected. These failure modes at high and low stresses have been categorized as regime I and regime III in the literature [7]. In general, the simulation results for SCC demonstrate similar failure mechanisms that are observed in regime I and regime III.

The crack propagation phase is defined as the duration from crack initiation to the instant that failure criterion is reached. The simulation results show that when the stress is high in regime III, the crack propagation phase is relatively short. This occurs as a result of more pronounced material creep. In addition, most simulations end by the strain limit criterion except one: $0.44 \mathrm{MPa}$ at $0.0005 \mathrm{ppm}$. In this case, the average fracture toughness of the ligament is less than $1 \%$ of its virgin state when failure occurs, and the energy flux into the crack tip is greater than the degraded fracture energy, which causes unstable crack growth under creep loading. The simulated crack propagation phase has also been compared with the published lifetime 
data. Colin et al. [7] provided the failure times of PE water pipes from south France, which varies from 5 to 30 years under various stresses (from 1 to $6 \mathrm{MPa}$ approximately). These lifetime data are comparable with the crack propagation phase predicted for $[\mathrm{DOC}]=0.15 \mathrm{ppm}$.

In summary, the current simulation framework is capable to predict the failure of polyethylene under two loading conditions: monotonic and creep loading. Specifically, under creep loading, SCC has been successfully simulated via a coupled simulation approach involving chemical reactions and diffusion calculations. Two failure mechanisms under SCC have been predicted: regime I primarily due to material creep, and regime III mainly controlled by oxidation reactions and diffusion.

\section{Conclusions}

In this paper, a novel co-simulation framework has been constructed to investigate the time-dependent crack propagation in a viscoplastic polymer. The simulation approach incorporates the chemical kinetics calculations from Matlab into finite element analysis in Abaqus. Two sets of numerical studies were performed: fracture of undegraded HDPE under monotonic loading, and stress corrosion cracking of HDPE in a chlorinated environment under creep loading. In fact, due to the generic functionality of Abaqus, this numerical framework is very flexible and readily adaptable for other materials and loading conditions.

A viscoplastic constitutive model is applied to characterize the time-dependent stress and strain relation. Its parameters have been calibrated through tensile testing in a previous study. Cohesive zone model with linear softening is utilized. Reaction induced $M_{w}$ loss, and subsequently the degradation of material strength and toughness, are captured via CZM: the traction separation relation is modified to be a function of $M_{w}$. Chemical kinetics and diffusion simulation is carried out in situ with FEA, to predict the $M_{w}$ profile in the specimen as the crack tip progresses. Two failure modes are considered to determine the lifetime: failure due to large strain where a strain limit of $15 \%$ is considered as a conservative criterion, and catastrophic unstable crack growth.

Results show that the failure mode in SCC is dependent on the applied load. At high stresses, the strain limit is quickly reached prior to the initiation of crack. This type of failure corresponds to the regime I on a lifetime-stress plot. At low stresses, chemical reaction tremendously corrodes the material properties over 
a longer time span. Therefore, failure is mainly caused by the loss of fracture property and, consequently, crack propagation. This failure mode is characteristic of the regime III brittle failure.

Two distinct crack propagation patterns have been identified due to difference in DOC concentration. At a high DOC concentration, corrosion is localized around the crack tip, and crack growth rate $d a / d t$ remains almost a constant throughout the entire failure history. By contrast, $d a / d t$ increases with the progression of crack when the DOC concentration is very low. This is caused by the dominance of chemical diffusion over the very long time span: material integrity in the remaining ligament is severely undermined. Thus, crack growth accelerates as a result of weakening material strength and toughness.

It should be pointed out that by using the current simulation framework, one can sufficiently explore the interactions of disinfectant concentration, loading, and even temperature in SCC analysis. The framework is also general enough to implement with other chemical reaction and diffusion models.

\section{References}

[1] L Audouin, V Langlois, J Verdu, and JCM De Bruijn. Role of oxygen diffusion in polymer ageing: kinetic and mechanical aspects. Journal of Materials science, 29(3):569-583, 1994.

[2] SRu Bodner and Y Partom. Constitutive equations for elastic-viscoplastic strain-hardening materials. Journal of Applied Mechanics, 42(2):385-389, 1975.

[3] Byoung-Ho Choi, Alexander Chudnovsky, and Kalyan Sehanobish. Stress corrosion cracking in plastic pipes: observation and modeling. International Journal of Fracture, 145(1):81-88, 2007.

[4] Byoung-Ho Choi, Zhenwen Zhou, Alexander Chudnovsky, Salvatore S Stivala, Kalyan Sehanobish, and Clive P Bosnyak. Fracture initiation associated with chemical degradation: observation and modeling. International Journal of Solids and Structures, 42(2):681-695, 2005.

[5] Alexander Chudnovsky, Zhenwen Zhou, Haiying Zhang, and Kalyan Sehanobish. Lifetime assessment of engineering thermoplastics. International Journal of Engineering Science, 59:108-139, 2012.

[6] Ozgen U Colak and Necmi Dusunceli. Modeling viscoelastic and viscoplastic behavior of high density polyethylene (hdpe). Journal of Engineering Materials and Technology, 128(4):572-578, 2006. 
[7] Xavier Colin, Ludmila Audouin, Jacques Verdu, Magali Rozental-Evesque, Benjamin Rabaud, Florencio Martin, and Francis Bourgine. Aging of polyethylene pipes transporting drinking water disinfected by chlorine dioxide. part iilifetime prediction. Polymer Engineering \& Science, 49(8):1642-1652, 2009.

[8] Alberto Corigliano, Stefano Mariani, and Anna Pandolfi. Numerical analysis of rate-dependent dynamic composite delamination. Composites Science and Technology, 66(6):766-775, 2006.

[9] JP Dear and NS Mason. Effect of chlorine on polyethylene pipes in water distribution networks. Proceedings of the Institution of Mechanical Engineers, Part L: Journal of Materials Design and Applications, 220(3):97-111, 2006.

[10] Clémence Devilliers, B Fayolle, Lucien Laiarinandrasana, S Oberti, and E Gaudichet-Maurin. Kinetics of chlorine-induced polyethylene degradation in water pipes. Polymer degradation and stability, 96(7):1361-1368, 2011.

[11] AD Drozdov et al. Viscoelasticity and viscoplasticity of semicrystalline polymers: Structure-property relations for high-density polyethylene. Computational materials science, 39(4):729-751, 2007.

[12] Donald E. Duvall and Dale B Edwards. Field failure mechanisms in hdpe potable water pipe. In Society of Plastic Engineers ANTEC 2011.

[13] M Elices, GV Guinea, J Gomez, and J Planas. The cohesive zone model: advantages, limitations and challenges. Engineering fracture mechanics, 69(2):137-163, 2002.

[14] Hanxiao Ge, Haiyan Li, and Susan C. Mantell. Modeling polymer failure under creep loading through simulation of crack growth. In Society of Plastic Engineers ANTEC 2014.

[15] H Ben Hadj Hamouda, Lucien Laiarinandrasana, and Roland Piques. Viscoplastic behaviour of a medium density polyethylene (mdpe): Constitutive equations based on double nonlinear deformation model. International journal of plasticity, 23(8):1307-1327, 2007.

[16] YG Hsuan, RM Koerner, and AE Lord Jr. Stress-cracking resistance of high-density polyethylene geomembranes. Journal of geotechnical engineering, 119(11):1840-1855, 1993. 
[17] Yan-Ling Huang and Norman Brown. The effect of molecular weight on slow crack growth in linear polyethylene homopolymers. Journal of materials science, 23(10):3648-3655, 1988.

[18] Pavel Hutař, Martin Ševčík, Luboš Náhlík, Gerald Pinter, Andreas Frank, and Ivaylo Mitev. A numerical methodology for lifetime estimation of hdpe pressure pipes. Engineering fracture mechanics, 78(17):3049-3058, 2011.

[19] SM Kurtz, CM Rimnac, TJ Santner, and DL Bartel. Exponential model for the tensile true stress-strain behavior of as-irradiated and oxidatively degraded ultra high molecular weight polyethylene. Journal of orthopaedic research, 14(5):755-761, 1996.

[20] HJ Kwon and P-YB Jar. New energy partitioning approach to the measurement of plane-strain fracture toughness of high-density polyethylene based on the concept of essential work of fracture. Engineering Fracture Mechanics, 74(16):2471-2480, 2007.

[21] R W Lang, A Stern, and G Doerner. PREDICTION MODELS FOR THERMOPLASTICS PIPES UNDER INTERNAL PRESSURE. 247:131-145, 1997.

[22] Hongtao Liu, Maria Anna Polak, and Alexander Penlidis. A practical approach to modeling timedependent nonlinear creep behavior of polyethylene for structural applications. Polymer Engineering \& Science, 48(1):159-167, 2008.

[23] Xici Lu and Norman Brown. The ductile-brittle transition in a polyethylene copolymer. Journal of materials science, 25(1):29-34, 1990.

[24] Spandan Maiti and Philippe H Geubelle. A cohesive model for fatigue failure of polymers. Engineering Fracture Mechanics, 72(5):691-708, 2005.

[25] Vigdis Olden, Christian Thaulow, Roy Johnsen, Erling Østby, and Torodd Berstad. Influence of hydrogen from cathodic protection on the fracture susceptibility of $25 \%$ cr duplex stainless steel-constant load sent testing and fe-modelling using hydrogen influenced cohesive zone elements. Engineering Fracture Mechanics, 76(7):827-844, 2009.

[26] Anita Redhead, Andreas Frank, and Gerald Pinter. Accelerated characterization of the resistance against crack initiation and slow crack growth of polyethylene for geomembrane application under the influence of media. In Society of Plastic Engineers ANTEC 2012. 
[27] AS Saleemi and JA Nairn. The plane-strain essential work of fracture as a measure of the fracture toughness of ductile polymers. Polymer Engineering \& Science, 30(4):211-218, 1990.

[28] Seong Hyeok Song, Glaucio H Paulino, and William G Buttlar. Simulation of crack propagation in asphalt concrete using an intrinsic cohesive zone model. Journal of Engineering Mechanics, 132(11):1215-1223, 2006.

[29] Chuntao Zhang and Ian D Moore. Nonlinear mechanical response of high density polyethylene. part ii: Uniaxial constitutive modeling. Polymer Engineering \& Science, 37(2):414-420, 1997. 